\title{
Successions in words and compositions
}

\author{
Arnold Knopfmacher ${ }^{\dagger}$, Augustine Munagi ${ }^{\dagger}$ and Stephan Wagner $^{\ddagger}$
}

September 3, 2009

\begin{abstract}
†The John Knopfmacher Centre for Applicable Analysis and Number Theory, School of Mathematics, University of the Witwatersrand, Johannesburg, South Africa

‡Department of Mathematical Sciences, Stellenbosch University, 7602 Stellenbosch, South

Africa.

${ }^{\dagger}$ Arnold.Knopfmacher@wits.ac.za, ${ }^{\dagger}$ augustine.munagi@wits.ac.za, ${ }^{\ddagger}$ swagner@sun.ac.za
\end{abstract}

\begin{abstract}
We consider words over the alphabet $[k]=\{1,2, \ldots, k\}, k \geq 2$. For a fixed nonnegative integer $p$, a $p$-succession in a word $w_{1} w_{2} \cdots w_{n}$ consists of two consecutive letters of the form $\left(w_{i}, w_{i}+p\right), i=1,2, \ldots, n-1$. We analyze words with respect to a given number of contained $p$-successions. First we find the mean and variance of the number of $p$-successions. We then determine the distribution of the number of $p$-successions in words of length $n$ as $n$ (and possibly $k$ ) tends to infinity; a simple instance of a phase transition (Gaussian-Poisson-degenerate) is encountered. Finally we also investigate successions in compositions of integers.
\end{abstract}

\section{Introduction}

We consider words over the alphabet $[k]=\{1,2, \ldots, k\}, k \geq 2$. For a fixed nonnegative integer $p$, a $p$-succession in a word $w_{1} w_{2} \cdots w_{n}$ consists of two consecutive letters of the form $\left(w_{i}, w_{i}+p\right), i=1,2, \ldots, n-1$. For example the word 1324122243 contains 3 instances of 2-successions: $13,24,24$. It is immediate that if $p \geq k$, then no word over $[k]$ can contain a $p$-succession.

In this paper we analyze words with respect to a given number of contained $p$-successions. We will also investigate successions in compositions (ordered partitions) of integers.

The subject of enumeration of finite sequences according to the number of $p$-successions has been much studied in the literature. The classical definition, in which $p=1$, was first applied by Kaplansky and Riordan, in the 1940's, to the enumeration of subsets of $[k]$ (see $[9,17])$. Subsequently, several authors have considered the enumeration of permutations of $[k]$ by the number of 1-successions, in conjunction with other well-known permutation statistics $[4,15,16,20]$. Extentions of the 1-succession idea in the case of subsets and set partitions have been studied in $[11]$ and $[12,13]$, respectively. Recently, two of the 
authors have carried out an interesting enumeration of integer partitions with respect to $p$-successions in [10].

Patterns in words, of which successions are a special case, have also been studied extensively in the past, also in view of their importance in computer science. A general framework for the analysis of patterns was developed in the late seventies and early eighties, in particular in the works of Goulden and Jackson [6] and Guibas and Odlyzko [7]. Nowadays, there are even software packages available that determine generating functions for the problem of counting occurrences of patterns in words automatically, see [1, 14]. For further information on this rich subject, we refer to the books by Flajolet and Sedgewick [5] and Szpankowski [19] and the references therein.

We are interested in the distribution of the number of $p$-successions in words of length $n$ as $n$ (and possibly $k$ ) tends to infinity. To this end, we first derive a bivariate generating function for the number of words with a given number of $p$-successions in Section 2. The limiting distribution is obtained in Section 3, see Theorem 3; it is a well known fact that the distibution of the number of certain pattern occurrences is asymptotically Gaussian [2] as $n \rightarrow \infty$ if $k$, the size of the alphabet, is fixed. If $n$ and $k$ are allowed to grow simultaneously, it turns out that this remains true as long as $n$ grows faster than $k$. If $k$ grows at the same speed as $k$, however, we encounter a phase transition: the limiting distribution is a Poisson distribution in this case. For even larger $k$, the distribution becomes degenerate.

In Section 4 we determine asymptotics for words with no $p$-successions. The enumeration of integer compositions by the number of $p$-successions is considered in Section 5 . The asserted results include the mean and variance of the number of $p$-successions in a random composition of an integer $n$. Again, the limiting distribution is found to be Gaussian.

\section{Generating functions}

We denote the length of a word $w$ by $\ell(w)$, the last letter of $w$ by $t(w)$ and the number of its $p$-successions by $s(w) ; p$ is assumed to be fixed throughout the paper, hence we ignore the dependence of $s(w)$ on $p$. Furthermore, we will also assume that $p$ is nonnegative, since a $p$-succession in a word $w$ corresponds to a $(-p)$-succession in the reversed word. Finally, we assume that $k>p$, since otherwise there cannot be any $p$-succession in any word over the alphabet $[k]$. Define the generating function

$$
v_{j}(x, y)=\sum_{w: t(w)=j} x^{\ell(w)} y^{s(w)}
$$

where the summation is over all words whose last letter is $j(j \in[k])$. It is easy to see that the functions $v_{1}, v_{2}, \ldots, v_{k}$ satisfy the functional equation

$$
v_{j}(x, y)= \begin{cases}x+x \sum_{i=1, i \neq j-p}^{k} v_{i}(x, y)+x y v_{j-p}(x, y) & j>p, \\ x+x \sum_{i=1}^{k} v_{i}(x, y) & j \leq p .\end{cases}
$$

Assume first that $p>0$; write $k=a p+b$, where $0 \leq b<p$, and set $V(x, y)=1+$ $\sum_{i=1}^{k} v_{i}(x, y)$. Then $V(x, y)$ is the generating function for all words (including the empty 
word), which is what we are actually interested in. It follows that $v_{j}(x, y)=x V(x, y)$ for $j \leq p$ and

$$
v_{j}(x, y)=x V(x, y)+x(y-1) v_{j-p}(x, y)
$$

otherwise. Straightforward induction yields

$$
v_{j}(x, y)=\frac{1-x^{r}(y-1)^{r}}{1-x(y-1)} \cdot x V(x, y)
$$

if $(r-1) p<j \leq r p$. Writing $z=x(y-1)$ for convenience, we can rewrite the sum of all $v_{j}$ as follows:

$$
\begin{aligned}
V(x, y) & =1+\sum_{i=1}^{k} v_{i}(x, y)=1+p x V(x, y) \sum_{r=1}^{a} \frac{1-z^{r}}{1-z}+b x V(x, y) \frac{1-z^{a+1}}{1-z} \\
& =1+\frac{x V(x, y)}{1-z}\left(a p-\frac{p z\left(1-z^{a}\right)}{1-z}+b\left(1-z^{a+1}\right)\right) \\
& =1+\frac{x V(x, y)}{1-z}\left(k-\frac{z}{1-z}\left(p\left(1-z^{a}\right)+b(1-z) z^{a}\right)\right) .
\end{aligned}
$$

Solving for $V(x, y)$ yields

$$
\begin{aligned}
V(x, y) & =\left(1-\frac{x}{(1-z)^{2}}\left(k(1-z)-z\left(p\left(1-z^{a}\right)+b(1-z) z^{a}\right)\right)\right)^{-1} \\
& =\left(1-\frac{x}{(1-z)^{2}}\left(k-(k+p) z+(p-b) z^{a+1}+b z^{a+2}\right)\right)^{-1}
\end{aligned}
$$

The special case $p=1$ occurs as Exercise 2.4.14 in [6]. The case $p=0$ can be treated in a similar way, and indeed one obtains the same formula (with $p=b=0$, even though $a$ is undefined in this case). Then the generating function simply reduces to

$$
V(x, y)=\left(1-\frac{k x}{1-z}\right)^{-1}
$$

It should also be noted that

$$
V(x, 1)=\frac{1}{1-k x},
$$

as expected. Differentiating the generating function with respect to $y$ and plugging in $y=1$, one immediately finds explicit formulae for the mean and variance of the number of successions: one has

and

$$
V_{y}(x, 1)=\frac{(k-p) x^{2}}{(1-k x)^{2}}
$$

$$
V_{y y}(x, 1)+V_{y}(x, 1)=\frac{2(k-p)^{2} x^{4}}{(1-k x)^{3}}+\frac{(k-p) x^{2}}{(1-k x)^{2}}+[a>1] \frac{2(k-2 p) x^{3}}{(1-k x)^{2}} .
$$

Here we use Iverson's notation: $[P]=1$ if $P$ is true and $[P]=0$ otherwise. Extracting coefficients and noting that $[a>1]=[k \geq 2 p]$, one obtains the following theorem: 
Theorem 1 The average number of p-successions in words of length $n$ is

$$
\frac{(k-p)(n-1)}{k^{2}}
$$

for $n>0$, while the variance is given by

$$
\frac{(k-p)(n-1)}{k^{2}}-\frac{(k-p)^{2}(3 n-5)}{k^{4}}+[k \geq 2 p] \frac{2(k-2 p)(n-2)}{k^{3}}
$$

for $n>1$.

\section{Limiting distribution}

Let us now consider the distribution of the number of $p$-successions in more detail. If $k$ is constant, then it follows easily from general theorems that the limiting distribution is Gaussian. Actually, this is known in more generality for arbitrary patterns in words [2], we also refer to [5, Note IX.33] and the references therein. Therefore, we consider a more general model in which $k$, the size of the alphabet, grows simultaneously with the length of our random words. It turns out that we have a very simple example of a phase transition: if $k$ grows slowly compared to $n$ (so that $\frac{k}{n} \rightarrow 0$ ), the limiting distribution is still Gaussian. If, on the other hand, $\frac{k}{n} \rightarrow \infty$, then Theorem 1, together with the Markov inequality, shows that the number of $p$-successions is almost surely 0 . In the remaining case that $k$ and $n$ are of the same asymptotic order, we will obtain a Poisson distribution in the limit.

In order to prove these results, we return to our bivariate generating function. For the distribution of the number of successions, the behavior around $y=1$ (and thus $z=0$ ) is essential. First we prove the following lemma:

Lemma 2 If $|y-1| \leq \frac{1}{10}$, then the polynomial

$P(x)=(1-x(y-1))^{2}-x\left(k-(k+p) x(y-1)+(p-b) x^{a+1}(y-1)^{a+1}+b x^{a+2}(y-1)^{a+2}\right)$

has exactly one zero $\rho=\rho(u, k)$ such that $|\rho|<\frac{2}{k}$, where $u=y-1$. This zero satisfies the inequality

$$
\left|\rho-\frac{1}{k}\right| \leq \frac{13|u|}{k^{2}} .
$$

Proof: We compare the polynomial to the linear polynomial $1-k x$, which clearly has exactly one zero inside the circle $|x|=\frac{2}{k}$. On this circle, one has $|1-k x| \geq 1$ and on the other hand, writing $u=y-1$ (so that $|u| \leq \frac{1}{10}$ ),

$$
\begin{aligned}
|P(x)-(1-k x)| & =\left|-2 x u+x^{2} u^{2}+(k+p) x^{2} u-(p-b) x^{a+2} u^{a+1}-b x^{a+3} u^{a+2}\right| \\
& \leq 2|x||u|+|x|^{2}|u|^{2}+(k+p)|x|^{2}|u|+(p-b)|x|^{a+2}|u|^{a+1}+b|x|^{a+3}|u|^{a+2} \\
& \leq 2|x||u|+|x|^{2}|u|^{2}+2 k|x|^{2}|u|+p|x|^{3}|u|^{2} \\
& \leq \frac{2}{5 k}+\frac{1}{25 k^{2}}+\frac{4}{5 k}+\frac{2}{25 k^{2}}=\frac{6}{5 k}+\frac{3}{25 k^{2}}<1 .
\end{aligned}
$$


Hence, by Rouché's Theorem, there must be exactly one zero inside the circle $|x|=\frac{2}{k}$. Furthermore, the above derivation shows that

$$
|P(x)-(1-k x)| \leq \frac{12|u|}{k}+\frac{12|u|^{2}}{k^{2}} \leq \frac{13|u|}{k}
$$

holds for $|x| \leq \frac{2}{k}$. Hence, if $P(\rho)=0$, one has

$$
|1-k \rho| \leq \frac{13|u|}{k}
$$

and thus

$$
\left|\rho-\frac{1}{k}\right| \leq \frac{13|u|}{k^{2}}
$$

as claimed.

Now we can apply the residue theorem to extract the coefficient of $x^{n}$ from $V(x, y)$ : if $|u|=|y-1| \leq \frac{1}{10}$, then

$$
\left[x^{n}\right] V(x, y)=\frac{1}{2 \pi i} \oint_{|z|=r} z^{-n-1} V(z, y) d y=\frac{1}{2 \pi i} \oint_{|z|=r} z^{-n-1}(1-z(y-1))^{2} P(z)^{-1} d y
$$

for any $0<r<|\rho|$. We shift the path of integration to obtain

$$
\begin{aligned}
{\left[x^{n}\right] V(x, y) } & =-\rho^{-n-1} \operatorname{Res}_{z=\rho}(1-u z)^{2} P(z)^{-1}+\frac{1}{2 \pi i} \oint_{|z|=2 / k} z^{-n-1}(1-u z)^{2} P(z)^{-1} d y \\
& =-\frac{\rho^{-n}(1-u \rho)^{2}}{\rho P^{\prime}(\rho)}+\frac{1}{2 \pi i} \oint_{|z|=2 / k} z^{-n-1}(1-u z)^{2} P(z)^{-1} d y
\end{aligned}
$$

By the inequalities above, $|P(z)|$ is uniformly bounded below on the circle $|z|=\frac{2}{k}$ by an absolute positive constant. Hence,

$$
\left[x^{n}\right] V(x, y)=-\frac{\rho^{-n}(1-u \rho)^{2}}{\rho P^{\prime}(\rho)}+O\left((k / 2)^{n}\right),
$$

uniformly for $|u| \leq \frac{1}{10}$. Note that $\rho \leq \frac{1}{k}+\frac{13|u|}{k^{2}} \leq \frac{33}{20 k}$, so the error term is indeed smaller than the main term by an exponential factor. For fixed $k$, this formula would already imply a central limit theorem by Hwang's Quasi-Power Theorem ([8], see also [5, Theorem IX.8]). If $k$ is allowed to grow with $n$, we have to do a little more work.

First we need more precise asymptotic information about $\rho$ : we assume that $a>1$, since the case $a=1$ can be treated analogously and since it can only occur if $k$ is bounded. Noting that $\rho=O\left(k^{-1}\right)$, the definition of $\rho$ yields

$$
0=P(\rho)=1-(k+2 u) \rho+\left(u^{2}+k u+p u\right) \rho^{2}+O\left(k^{-4} u^{3}\right)
$$


and thus

$$
\begin{aligned}
\rho & =\frac{k+2 u-\sqrt{(k+2 u)^{2}-4\left(u^{2}+k u+p u\right)\left(1+O\left(k^{-4} u^{3}\right)\right)}}{2\left(u^{2}+k u+p u\right)} \\
& =\frac{k+2 u-\sqrt{k^{2}-4 p u+O\left(k^{-3} u^{4}\right)}}{2 u(k+p+u)} \\
& =\frac{1}{k}-\frac{k-p}{k^{3}} u+\frac{k^{2}-2 k p+2 p^{2}}{k^{5}} u^{2}+O\left(k^{-4} u^{3}\right)
\end{aligned}
$$

after a few simplifications. Plugging in, one also obtains

$$
-\frac{(1-u \rho)^{2}}{\rho P^{\prime}(\rho)}=1+O\left(k^{-1} u\right)
$$

Let $\omega_{n}$ denote the number of $p$-successions in a random word of length $n$. The moment generating function of this random variable is given by

$$
\mathbb{E}\left(e^{\omega_{n} t}\right)=k^{-n}\left[x^{n}\right] V\left(x, e^{t}\right) .
$$

Instead of dealing with $\omega_{n}$ directly, we consider the normalized random variable $\varpi_{n}=$ $\frac{\omega_{n}-\mu_{n}}{\sigma_{n}}$, where $\mu_{n}$ and $\sigma_{n}^{2}$ are the mean and variance of $\omega_{n}$ respectively, as given in Theorem 1 . The moment generating function of $\varpi_{n}$ is given by

$$
\mathbb{E}\left(e^{\left(\omega_{n}-\mu_{n}\right) t / \sigma_{n}}\right)=k^{-n} e^{-\mu_{n} t / \sigma_{n}}\left[x^{n}\right] V\left(x, e^{t / \sigma_{n}}\right) .
$$

Now we apply the asymptotic formula for $\left[x^{n}\right] V(x, y)$ with $y=e^{t / \sigma_{n}}$ (and thus $u=y-1=$ $\left.\frac{t}{\sigma_{n}}+\frac{t^{2}}{2 \sigma_{n}^{2}}+O\left(\frac{t^{3}}{\sigma_{n}^{3}}\right)\right)$ to obtain

$$
\rho=\frac{1}{k}-\frac{k-p}{k^{3}} \cdot \frac{t}{\sigma_{n}}+\left(\frac{k^{2}-2 k p+2 p^{2}}{k^{5}}-\frac{k-p}{2 k^{3}}\right) \cdot \frac{t^{2}}{\sigma_{n}^{2}}+O\left(\frac{t^{3}}{k^{2} \sigma_{n}^{3}}\right) .
$$

It follows that

$$
\log (k \rho)=-\frac{k-p}{k^{2}} \cdot \frac{t}{\sigma_{n}}-\frac{k^{3}-k^{2} p-k^{2}+2 k p-3 p^{2}}{2 k^{4}} \cdot \frac{t^{2}}{\sigma_{n}^{2}}+O\left(\frac{t^{3}}{k \sigma_{n}^{3}}\right)
$$

and thus

$$
\begin{aligned}
\mathbb{E} & \left(e^{\left(\omega_{n}-\mu_{n}\right) t / \sigma_{n}}\right) \\
= & k^{-n} e^{-\mu_{n} t / \sigma_{n}}\left[x^{n}\right] V\left(x, e^{t / \sigma_{n}}\right)=e^{-\mu_{n} t / \sigma_{n}} \cdot\left(-\frac{(1-u \rho)^{2}}{\rho P^{\prime}(\rho)}\right) \cdot(k \rho)^{-n}+O\left(e^{-\mu_{n} t / \sigma_{n}} 2^{-n}\right) \\
= & \exp \left(-\frac{\mu_{n} t}{\sigma_{n}}+\frac{(k-p) n}{k^{2}} \cdot \frac{t}{\sigma_{n}}+\frac{\left(k^{3}-k^{2} p-k^{2}+2 k p-3 p^{2}\right) n}{2 k^{4}} \cdot \frac{t^{2}}{\sigma_{n}^{2}}+O\left(\frac{t^{3} n}{k \sigma_{n}^{3}}\right)\right) \\
& \cdot\left(1+O\left(\frac{t}{k \sigma_{n}}\right)\right)+O\left(e^{-\mu_{n} t / \sigma_{n}} 2^{-n}\right) .
\end{aligned}
$$


Taking into account that

$$
\mu_{n}=\frac{(k-p) n}{k^{2}}+O\left(k^{-1}\right) \quad \text { and } \quad \sigma_{n}^{2}=\frac{\left(k^{3}-k^{2} p-k^{2}+2 k p-3 p^{2}\right) n}{k^{4}}+O\left(k^{-1}\right),
$$

this reduces to

$$
\begin{aligned}
\mathbb{E}\left(e^{\left(\omega_{n}-\mu_{n}\right) t / \sigma_{n}}\right) & =\exp \left(\frac{t^{2}}{2}+O\left(\frac{t}{k \sigma_{n}}+\frac{t^{3} n}{k \sigma_{n}^{3}}\right)\right)+O\left(e^{-\mu_{n} t / \sigma_{n}} 2^{-n}\right) \\
& =\exp \left(\frac{t^{2}}{2}+O\left(\frac{t}{\sqrt{k n}}+\frac{t^{3} \sqrt{k}}{\sqrt{n}}\right)\right)+O\left(e^{-\mu_{n} t / \sigma_{n}} 2^{-n}\right) .
\end{aligned}
$$

Hence, the moment generating function tends to $e^{t^{2} / 2}$ (pointwise and uniformly on compact subsets of $\mathbb{R}$ ) if $\sigma_{n}^{2} \sim \frac{n}{k} \rightarrow \infty$ (or, in other words, $\frac{k}{n} \rightarrow 0$ ), which is the moment generating function of a standard normal distribution. By Curtiss's Theorem [3], this implies that the distribution of $\varpi_{n}$ tends weakly to a standard normal distribution.

Things are slightly different if $k$ is proportional to $n$, i.e. $k \sim \frac{n}{c}$ for some positive constant $c$. In this case, the mean and variance no longer tend to infinity; in fact, both tend to $c$. However, the proof of convergence to a limiting distribution is actually shorter in this case: one can even work directly with the ordinary probability function of $\omega_{n}$, namely

$$
\sum_{i=0}^{\infty} \mathbb{P}\left(\omega_{n}=i\right) y^{i}=k^{-n}\left[x^{n}\right] V(x, y) .
$$

We obtain the following asymptotic formulae:

$$
\rho=\frac{1}{k}-\frac{u}{k^{2}}+O\left(k^{-3}\right)
$$

thus

$$
\log (k \rho)=-\frac{u}{k}+O\left(k^{-2}\right)
$$

and

$$
-\frac{(1-u \rho)^{2}}{\rho P^{\prime}(\rho)}=1+O\left(k^{-1}\right)
$$

Hence we have

$$
\begin{aligned}
k^{-n}\left[x^{n}\right] V(x, y) & =\left(1+O\left(k^{-1}\right)\right)(k \rho)^{-n}+O\left(2^{-n}\right) \\
& =\left(1+O\left(k^{-1}\right)\right) \exp \left(\frac{u n}{k}+O\left(n k^{-2}\right)\right)+O\left(2^{-n}\right) \\
& =\exp \left(\frac{u n}{k}+O\left(n^{-1}\right)\right)+O\left(2^{-n}\right),
\end{aligned}
$$

which tends to $\exp (c u)=\exp (c(y-1))$ (at least if $\left.|u|<\frac{1}{10}\right)$, which is exactly the probability generating function of a Poisson distribution with mean and variance $c$. Using $[5$, Theorem IX.1], it follows that the distribution of $\omega_{n}$ tends to a Poisson distribution, and we end up with the following theorem that summarizes the results of this section: 
Theorem 3 If $\frac{k}{n} \rightarrow 0$, then the distribution of the number of p-successions is asymptotically normal; if $k$ and $n$ are of the same order, i.e., $k \sim \frac{n}{c}$ for some constant $c$, then the distribution of the number of p-successions tends to a Poisson distribution. Finally, if $\frac{k}{n} \rightarrow \infty$, then there are almost surely no p-successions in a random word of length $n$, so the distribution is degenerate in this case.

\section{Words without $p$-successions}

The generating function for words without $p$-successions can be found by putting $y=0$ in $V(x, y)$. One obtains

$$
W(x)=\left(1-\frac{x}{(1+x)^{2}}\left(k+(k+p) x+(p-b)(-x)^{a+1}+b(-x)^{a+2}\right)\right)^{-1} .
$$

The dominant pole of this function must lie between $\frac{1}{k}$ and $\frac{1}{k-1}$ : this follows from the observation that there are at least $k(k-1)^{n-1}$ words of length $n$ without $p$-successions, but at most $k^{n}$ such words. For large $k$, this pole (let us denote it by $\rho_{0}$ ) can be approximated quite well: one has

$$
\left|(p-b)\left(-\rho_{0}\right)^{a+2}+b\left(-\rho_{0}\right)^{a+3}\right| \leq p \rho_{0}^{k / p+1}=O\left((k-1)^{-k / p-1}\right)
$$

and thus

$$
\left(1+\rho_{0}\right)^{2}-k \rho_{0}-(k+p) \rho_{0}^{2}+O\left((k-1)^{-k / p-1}\right)=0,
$$

from which one deduces

$$
\rho_{0}=\frac{\sqrt{k^{2}+4 p}-(k-2)}{2(k+p-1)}+O\left((k-1)^{-k / p-2}\right) .
$$

The coefficient $\left[x^{n}\right] W(x)$ is asymptotically $\left(-\operatorname{Res}_{z=\rho_{0}} W(z)\right) \rho_{0}^{-n-1}$, and so one obtains the following theorem:

Theorem 4 The number of words of length $n$ without p-successions is asymptotically given by $\alpha_{k} \beta_{k}^{n}$, where

$$
\alpha_{k}=\frac{(k+p)\left(k+\sqrt{k^{2}+4 p}\right)+2 p}{2(k+p-1) \sqrt{k^{2}+4 p}}+O\left((k-1)^{-k / p-1}\right)
$$

and

$$
\beta_{k}=\frac{\sqrt{k^{2}+4 p}+k-2}{2}+O\left((k-1)^{-k / p}\right) .
$$

Note that the formulae for $\alpha_{k}$ and $\beta_{k}$ are exact (without the error term) if $p=0$. 


\section{Successions in compositions}

Compositions can be treated in a similar way: in analogy to Section 2, we define the generating function $v_{j}(x, y)$ for compositions whose last summand is $j$ (this approach is essentially equivalent to the "adding a slice" technique, see [5, Section 3.7]). The functions $v_{1}, v_{2}, \ldots$ satisfy the functional equations

$$
v_{j}(x, y)= \begin{cases}x^{j}+x^{j} \sum_{i \geq 1, i \neq j-p} v_{i}(x, y)+x^{j} y v_{j-p}(x, y) & j>p, \\ x^{j}+x^{j} \sum_{i \geq 1} v_{i}(x, y) & j \leq p .\end{cases}
$$

We are interested in the combined generating function $V(x, y)=1+\sum_{j \geq 1} v_{j}(x, y)$ again. In order to find an expression for this function, we first introduce auxiliary functions $U_{r}(x, y)=\sum_{j \geq 1} x^{r j} v_{j}(x, y)$. Then $U_{0}(x, y)=V(x, y)-1$, and the functional equations stated above imply

$$
\begin{aligned}
U_{r}(x, y) & =\sum_{j \geq 1} x^{r j} v_{j}(x, y)=\sum_{j \geq 1} x^{r j} \cdot x^{j} V(x, y)+\sum_{j>p} x^{r j}(y-1) x^{j} v_{j-p}(x, y) \\
& =\frac{x^{r+1}}{1-x^{r+1}} \cdot V(x, y)+x^{(r+1) p}(y-1) \sum_{j \geq 1} x^{(r+1) j} v_{j}(x, y) \\
& =\frac{x^{r+1}}{1-x^{r+1}} \cdot V(x, y)+x^{(r+1) p}(y-1) U_{r+1}(x, y) .
\end{aligned}
$$

Substituting $x^{r(r+1) p / 2}(y-1)^{r} U_{r}(x, y)=T_{r}(x, y)$, one obtains

$$
T_{r}(x, y)=\frac{x^{r(r+1) p / 2+(r+1)}(y-1)^{r}}{1-x^{r+1}} \cdot V(x, y)+T_{r+1}(x, y)
$$

with $T_{0}(x, y)=U_{0}(x, y)=V(x, y)-1$ and thus by induction

$$
T_{r}(x, y)=V(x, y)-1-\left(\sum_{j=1}^{r}(y-1)^{j-1} \frac{x^{j(j-1) p / 2+j}}{1-x^{j}}\right) V(x, y)
$$

As $r \rightarrow \infty, T_{r}(x, y) \rightarrow 0$ (as a formal power series), and so we have

$$
V(x, y)=\left(1-\sum_{j=1}^{\infty}(y-1)^{j-1} \frac{x^{j(j-1) p / 2+j}}{1-x^{j}}\right)^{-1} .
$$

Note that one has $V(x, 1)=\frac{1-x}{1-2 x}$, as it should be. Furthermore, one can easily determine the first and second derivative in order to find the mean and variance:

$$
V_{y}(x, 1)=\frac{(1-x) x^{p+2}}{(1+x)(1-2 x)^{2}}
$$

and

$$
V_{y y}(x, 1)=\frac{2 x^{2 p+4}(1-x)}{(1+x)^{2}(1-2 x)^{3}}+\frac{2 x^{3 p+3}(1-x)}{\left(1+x+x^{2}\right)(1-2 x)^{2}} .
$$

Now one can read off the coefficients to obtain the following theorem: 
Theorem 5 The mean and variance of the number of p-successions in a random composition of $n$ are given by

$$
\mu_{n}=2^{-p}\left(\frac{n}{6}-\frac{3 p-1}{18}\right)+\frac{4}{9}(-1)^{p}\left(-\frac{1}{2}\right)^{n}
$$

for $n>p$ and

$$
\begin{aligned}
\sigma_{n}^{2}= & \left(\frac{2^{-p}}{6}-\frac{(6 p+7) 2^{-2 p}}{108}+\frac{2^{-3 p}}{7}\right) n \\
& -\left(\frac{(3 p-1) 2^{-p}}{18}-\frac{\left(27 p^{2}+36 p-19\right) 2^{-2 p}}{324}+\frac{(21 p+3) 2^{-3 p}}{49}\right)+O\left(n 2^{-n}\right) .
\end{aligned}
$$

Furthermore, the distribution of the number of p-successions is asymptotically normal.

Proof: The mean and variance follow directly from the explicit formulae for the derivatives of $V(x, y)$, so it remains to prove the limit law. This, however, is essentially a consequence of the fact that $V(x, y)$ is the quotient of two analytic functions (within suitable regions); see [5, Theorem IX.9].

It should be noted that an explicit formula for the variance can be given as well; since it is quite lengthy, only the main terms are provided here.

Remark It is interesting to compare the mean number of successions in compositions, which is linear in $n$, with the mean number of successions in partitions of integers, which is shown in [10] to grow like $\frac{\sqrt{\frac{6}{\pi^{2}}}}{p(p+1)} n^{1 / 2}$ as $n \rightarrow \infty$.

\section{Conclusion}

It is quite likely that all our results concerning limiting distributions, in particular the phase transition observed in Theorem 3, hold for more general patterns: if $\mathcal{S}(k)$ is a suitable collection of patterns in words over the alphabet $[k]$, and the size of $\mathcal{S}(k)$ grows linearly with $k$, then it is probable that the same type of phase transition occurs. The technical details might be intricate, though (in particular the proper definition of "suitable collection of patterns").

\section{References}

[1] F. Bassino, J. Clément, J. Fayolle and P. Nicodème, Counting occurrences for a finite set of words: an inclusion-exclusion approach, Discrete Math. Theor. Comput. Sci. Proc. (2007), Proceedings of the 2007 Conference on Analysis of Algorithms, pp. 2944. 
[2] E. A. Bender and F. Kochman, The distribution of subword counts is usually normal, European J. Combin. 14 (1993), no. 4, 265-275.

[3] J. H. Curtiss, A note on the theory of moment generating functions, Ann. Math. Statistics 13 (1942), 430-433.

[4] W. Dymacek, D. P. Roselle, Circular permutations by number of rises and successions, J. Combin. Theory Ser. A 25 (1978), no. 2, 196-201.

[5] P. Flajolet and R. Sedgewick, Analytic Combinatorics, Cambridge University Press, 2008.

[6] I. P. Goulden and D. M. Jackson, Combinatorial enumeration, Dover, 2004.

[7] L. J. Guibas and A. M. Odlyzko, String overlaps, pattern matching, and nontransitive games, J. Combin. Theory Ser. A 30 (1981), no. 2, 183-208.

[8] H.-K. Hwang, On convergence rates in the central limit theorems for combinatorial structures, European J. Combin. 19 (1998), 329-343.

[9] I. Kaplansky, Solution of the "problem des menages," Bull. Amer. Math. Soc. 49 (1943), 784-785.

[10] A. Knopfmacher, A. O. Munagi, Successions in integer partitions, Ramanujan J. 18 (3) (2009), 239-255.

[11] A. O. Munagi, Combinations with successions and Fibonacci numbers, Fibonacci Quart. 45.2 (2007) 104-114.

[12] A.O. Munagi, Extended Set Partitions with Successions, European J. Combin. 29(5) (2008) 1298-1308.

[13] A. O. Munagi, Set partitions with successions and separations, Int. J. Math. Math. Sci. 2005, no. 3, 451-463.

[14] J. Noonan and D. Zeilberger, The Goulden-Jackson cluster method: extensions, applications and implementations, J. Differ. Equations Appl. 5 (1999), no. 4-5, 355-377.

[15] J. W. Reilly, Counting permutations by successions and other figures, Discrete Math. 32 (1980), no. 1, 69-76.

[16] J. R. Reilly, S. M. Tanny, Counting successions in permutations, Stud. Appl. Math. 61 (1979), no. 1, 73-81.

[17] J. Riordan, Permutations without 3-sequences, Bull. Amer. Math. Soc. 51 (1945), $745-748$. 
[18] N. J. A. Sloane, (2006), The On-Line Encyclopedia of Integer Sequences, published electronically at http://www.research.att.com/njas/sequences/.

[19] W. Szpankowski, Average case analysis of algorithms on sequences, Wiley-Interscience, New York, 2001.

[20] S. M. Tanny, Permutations and successions, J. Combinatorial Theory Ser. A 21 (1976), no. 2, 196-202. 